\title{
On the Design of Turbo Packet Combining Schemes for Relay-Assisted Systems over Multi-Antenna Broadband Channels
}

\author{
Houda Chafnaji ${ }^{\ddagger *}$, Tarik Ait-Idir ${ }^{\ddagger *}$, Halim Yanikomeroglu ${ }^{+}$, and Samir Saoudi* \\ ${ }^{\ddagger}$ Communications Systems Department, INPT, Madinat Al Irfane, Rabat, Morocco \\ ${ }^{*}$ TELECOM Bretagne, Signal and Communications Department, CS 83818, 29238 Brest Cedex, France. \\ + Broadband Communications and Wireless Systems (BCWS) Centre, \\ Department of Systems and Computer Engineering, Carleton University, Ottawa, Canada \\ Emails: houda.chafnaji,samir.saoudi@telecom-bretagne.eu, aitidir@ieee.org, halim@sce.carleton.ca
}

\begin{abstract}
This paper focuses on turbo packet combining strategies for multi-relay-assisted systems operating over multiple-input-multiple-output (MIMO) broadband channel. We propose a frequency domain minimum mean square (MMSE)based turbo packet combining scheme where all slots received signals and their corresponding channel frequency responses (CFR)s are used to decode the data packet. We also provide an efficient recursive implementation way for the proposed scheme, and show that both its computational complexity and memory requirements are quite insensitive to the number of relays in the system. For the special case of cooperative automatic repeat request (ARQ) systems, we introduce an adaptive packet combining algorithm that enable to reduce the receiver implementation cost. Block error rate (BLER) performance are provided to demonstrate the gains offered by the proposed combining scheme over the conventional soft information-based combining.
\end{abstract}

Index Terms-Cooperative relaying, multiple-antenna systems, turbo equalization, packet combining.

\section{INTRODUCTION}

Relaying is an efficient diversity technique that allows to combat multi-path fading in wireless communications [1], [2]. In this transmission mechanism, one or more relays assist the communication between the source and destination to form a multiple- input-multiple-output (MIMO) system and therefore build up space-time diversity branches that are exploited at the destination. Several interesting relaying schemes have been proposed, among which are two basic modes: amplify-andforward (AF), and decode-and-forward (DF). The AF strategy represents the simplest way that a relay may cooperate with the source and the destination. Under this scheme, the relay simply amplifies the received signal and forwards it towards the destination. However, in the DF scheme, the relay first decodes the signal received from the source, re-encodes and retransmits it to the destination. This approach suffers from error propagation when the relay transmits an erroneously decoded data block [3]. Selective DF, where the relay only transmits when it can reliably decode the data packet, has been introduced as an efficient method to reduce error propagation [4].

To improve spatial diversity of a relaying system, signals received over the source-destination and the relay-destination links are combined at the receiver side. Most of the research work in this area has focused on flat fading channels. However, in practical systems, channels connecting the source, the relay and the destination may suffer from inter-symbol interference (ISI) caused by frequency-selective fading. In [5], the authors have introduced a packet combining strategy for AF scheme operating under the so-called protocol I, where the source broadcasts the data packet to both the relay and the destination during the first slot, and both the source and the relay re-send the packet to the destination during the second slot [6]. Block equalization has been proposed in [7] for protocol III, where the broadcast nature of the channel is not considered, i.e., the source sends to the relay during the first slot, and both the source and the relay send to the destination in the second slot. In [8], a frequency-domain equalizer with diversity combining has been proposed for single antenna cooperative systems with demodulate-and-forward relaying and using the so-called protocol II. In this protocol, the operation mode during the first slot is similar to that of protocol I, while during the second slot only the relay sends the packet to the destination.

In this paper, we consider a broadband multi-relay-assisted system using selective DF scheme. We focus on systems operating under the framework of protocol II as it is widely regarded as an efficient relaying scheme for increasing the overall throughput. In this paper, inspired by the packet combining concept introduced in [9], [10] for ARQ, we propose an MMSE-based turbo packet combining scheme for broadband relaying systems. We then provide an efficient recursive implementation for the proposed scheme, and show that both its computational complexity and memory requirements are quite insensitive to the number of relays in the system. We also introduce a low complexity adaptive packet combining scheme for the special case of cooperative ARQ communication, where the feedback from the destination is exploited and the packet repetition is activated only if the destination fails to decode the data packet [11], [12]. Throughout the paper we use the following notation: $(.)^{\top}$ and $(.)^{\mathrm{H}}$ are the transpose and the transpose conjugate of the argument, respectively. $\operatorname{diag}\{\mathbf{x}\}$ and $\operatorname{diag}\left\{\mathbf{X}_{1}, \cdots, \mathbf{X}_{m}\right\}$ denote the diagonal matrix and the block diagonal matrix constructed from $\mathbf{x} \in \mathbb{C}^{n}$ and from $\mathbf{X}_{1}, \cdots, \mathbf{X}_{m} \in \mathbb{C}^{n_{1} \times n_{2}}$, respectively. For $\mathbf{x} \in \mathbb{C}^{T N}$, $\mathbf{x}_{f}$ denotes the discrete Fourier transform (DFT) of $\mathbf{x}$, i.e. 
$\mathbf{x}_{f}=\mathbf{U}_{T, N} \mathbf{x}$, with $\mathbf{U}_{T, N}=\mathbf{U}_{T} \otimes \mathbf{I}_{N}$, where $\mathbf{I}_{N}$ is the $N \times N$ identity matrix, $\mathbf{U}_{T}$ is a unitary $T \times T$ matrix whose $(m, n)$ th element is $\left(\mathbf{U}_{T}\right)_{m, n}=\frac{1}{\sqrt{T}} e^{-j(2 \pi m n / T)}, j=\sqrt{-1}$, and $\otimes$ denotes the Kronecker product.

The remainder of the paper is organized as follows: In Section II, we introduce the relay system model. In Section III, we provide the structure of the proposed packet combining scheme as well as its efficient recursive implementation. The adaptive packet combining algorithm for cooperative ARQ systems is detailed in Section IV. The performance analysis is provided in Section V. Finally, the paper is concluded in Section VI.

\section{RELAY SYSTEM MODEL}

We consider a relay-assisted wireless communication system where the $M_{\mathrm{S}}$ antenna source denoted as $\mathrm{S}$ transmits information blocks to the $M_{\mathrm{D}}$ antenna destination denoted as $\mathrm{D}$ with the assistance of $K-1$ dedicated selective DF relays denoted as $\mathrm{R}_{2}, \cdots, \mathrm{R}_{k}, \cdots, \mathrm{R}_{K}$. Each relay $\mathrm{R}_{k}$ is equipped with $M_{\mathrm{R}_{k}}$ transmit and receive antennas. The sourcerelay ( $\mathrm{S} \rightarrow \mathrm{R}_{k}$ ), source-destination $(\mathrm{S} \rightarrow \mathrm{D})$, and relaydestination $\left(\mathrm{R}_{k} \rightarrow \mathrm{D}\right)$ links are assumed to be frequency selective. The channel matrices corresponding to the $A \rightarrow B$ link are $\mathbf{H}_{0}^{(A B)}, \cdots, \mathbf{H}_{L_{A B}-1}^{(A B)} \in \mathbb{C}^{M_{B} \times M_{A}}$, where $L_{A B}$ denotes the number of symbol-spaced taps, and $A \in\left\{\mathrm{S}, \mathrm{R}_{k}\right\}$, and $B \in\left\{\mathrm{R}_{k}, \mathrm{D}\right\}$. Their entries are zero-mean circularly symmetric complex Gaussian random variables. Cyclic prefix (CP)-aided transmission is assumed for all links. The average energies of the different links are $E_{\mathrm{SR}_{k}}, E_{\mathrm{SD}}$ and $E_{\mathrm{R}_{k} \mathrm{D}}$, and take into account the path-loss and shadowing effects of each link.

In this paper, the relay-assisted system use up to $K$ time slots for sending one information block from the source to the destination to guarantee orthogonal transmissions, where each slot spans $T$ channel use. First, the source encodes its data blocks using a space-time bit interleaved coded modulation (STBICM) encoder. The resulting symbol vector is given by,

$$
\mathbf{s} \triangleq\left[\mathbf{s}_{0}^{\top}, \cdots, \mathbf{s}_{T-1}^{\top}\right]^{\top} \in \mathcal{S}^{M_{S} T},
$$

where $\mathbf{s}_{i} \triangleq\left[s_{1, i}^{\top}, \cdots, s_{t, i}^{\top}, \cdots, s_{M_{\mathrm{S}}, i}^{\top}\right]^{\top} \in \mathcal{S}^{M_{\mathrm{S}}}$ is the symbol vector at channel use $i=0, \cdots, T-1$, and $\mathcal{S}$ is the symbol constellation set. During the first slot, the source inserts a $\mathrm{CP}$ symbol word of length $T_{C P}^{\mathrm{S} \rightarrow \mathrm{D}} \geq \max _{k=2, \cdots, K}\left(L_{\mathrm{SR}_{k}}, L_{\mathrm{SD}}\right)$, then broadcasts the resulting symbol frame to the $K-1$ relays and the destination. After $\mathrm{CP}$ deletion, the baseband $M_{\mathrm{D}} \times 1$ signal vector obtained at the destination is given by,

$$
\mathbf{y}_{i}^{(1)}=\sqrt{E_{\mathrm{SD}}} \sum_{l=0}^{L_{\mathrm{SD}}-1} \mathbf{H}_{l}^{(1)} \mathbf{s}_{(i-l) \bmod T}+\mathbf{n}_{i}^{(1)}
$$

where $\mathbf{H}_{l}^{(1)}=\mathbf{H}_{l}^{(\mathrm{SD})}$, and $\mathbf{n}_{i}^{(1)} \sim \mathcal{N}\left(\mathbf{0}_{M_{\mathrm{D} \times 1}}, \sigma^{2} \mathbf{I}_{M_{\mathrm{D}}}\right)$ is the thermal noise at the destination receiver. During the following $K-1$ slots, each relay decodes the signal received from the source, if the data packet is correctly decoded, the relay re-encodes and retransmits it to the destination during the allocated slot $k$, using $M_{\mathrm{S}}$ antennas, otherwise, the packet retransmission is not activated during slot $k$. At each slot $k=2, \ldots, K$, if the packet retransmission is activated, the $M_{\mathrm{D}} \times 1$ received signal vector at the destination side, after CP deletion, can be expressed as

$$
\mathbf{y}_{i}^{(k)}=\sqrt{E_{k}} \sum_{l=0}^{L_{k}-1} \mathbf{H}_{l}^{(k)} \mathbf{s}_{(i-l) \bmod T}+\mathbf{n}_{i}^{(k)} .
$$

where $\mathbf{H}_{l}^{(k)}=\mathbf{H}_{l}^{\left(\mathrm{R}_{k} \mathrm{D}\right)} \in \mathbb{C}^{M_{\mathrm{D}} \times M_{\mathrm{S}}}, L_{k}=L_{\mathrm{R}_{k} \mathrm{D}}$, and $E_{k}=$ $E_{\mathrm{R}_{k} \mathrm{D}}$.

\section{Frequency Domain MMSE-BAsed Turbo PACKet COMBINING}

In this paper the data packet is decoded in iterative fashion through the exchange of extrinsic information between the proposed soft packet combiner and SISO decoder. In cooperative ARQ systems, the destination performs the data packet decoding during each time slot $k$, while in fixed-relay based systems, the data packet decoding didn't start before the last time slot $K$. First, the frequency domain block signal vector $\underline{\mathbf{y}}_{f}^{(k)}$ is constructed. Second, the optimal soft combiner computes the extrinsic log-likelihood ratio (LLR) about coded and interleaved bits using a priori information. Then, the extrinsic LLRs corresponding to coded and interleaved bits are desinterleaved, and transferred to the SISO decoder. After a preset number of iterations, the decision about the data packet is performed. In cooperative ARQ systems, If the packet is incorrectly decoded at time slot $k$, a NACK message is sent to the relay $k+1$ which starts the packet retransmission process. If the packet is correctly decoded, the destination broadcast an ACK message to both source and relays to stop the relaying transmission and start the transmission of a new data packet during the next time slot. In this section, we propose an MMSE-based turbo packet combining scheme for broadband multi-relay-assisted systems. The concept performs, in the frequency domain, interference cancellation and MMSE filtering jointly for all slots by considering each slot as an additional set of virtual $M_{\mathrm{D}}$ receive antennas. We also introduce an efficient implementation scheme for systems with $K M_{D}>M_{S}$.

\section{A. The Proposed Turbo Packet Combining Scheme}

After $k$ time slots, the system (source, $k-1$ relays, and destination) can be viewed as a point to point MIMO link with $M_{\mathrm{S}}$ transmit and $k M_{\mathrm{D}}$ receive antennas. Using the "virtual antennas" concept, we construct the $k M_{\mathrm{D}} T \times 1$ block received signal vector $\underline{\mathbf{y}}^{(k)}$ after $k$ slots as,

$$
\underline{\mathbf{y}}^{(k)} \triangleq\left[\underline{\mathbf{y}}_{0}^{(k)^{\top}}, \cdots, \underline{\mathbf{y}}_{T-1}^{(k)^{\top}}\right]^{\top} \in \mathbb{C}^{k M_{\mathrm{D}} T},
$$

where

$$
\underline{\mathbf{y}}_{i}^{(k)} \triangleq\left[\mathbf{y}_{i}^{(1)^{\top}}, \cdots, \mathbf{y}_{i}^{(k)^{\top}}\right]^{\top} \in \mathbb{C}^{k M_{\mathrm{D}}}
$$

is the signal received over the $k M_{\mathrm{D}}$ virtual antennas corresponding to reception over $k$ consecutive slots using $M_{\mathrm{D}}$ receive antennas. The block communication model corresponding to this $k$-slot scheme is given by,

$$
\underline{\mathbf{y}}^{(k)}=\underline{\mathcal{H}}^{(k)} \mathbf{s}+\underline{\mathbf{n}}^{(k)},
$$


where $\underline{\mathcal{H}}^{(k)} \in \mathbb{C}^{k M_{\mathrm{D}} T \times M_{\mathrm{S}} T}$ is a block circulant matrix whose first $k M_{\mathrm{D}} T \times M_{\mathrm{S}}$ block column matrix is

$$
\left[\underline{\mathbf{H}}_{0}^{(\mathrm{k})^{\top}}, \cdots, \underline{\mathbf{H}}_{L-1}^{(\mathrm{k})^{\top}}, \mathbf{0}_{(T-L) k M_{\mathrm{D}} \times M_{\mathrm{S}}}\right]^{\top},
$$

with

$$
\begin{cases}L & =\max _{k=1, \cdots, K}\left(L_{k}\right), \\ \underline{\mathbf{H}}_{l}^{(k)} & \triangleq\left[\sqrt{E_{1}} \mathbf{H}_{l}^{(1)^{\top}}, \cdots, \sqrt{E_{k}} \mathbf{H}_{l}^{(k)^{\top}}\right]^{\top} \in \mathbb{C}^{k M_{\mathrm{D}} \times M_{\mathrm{S}}},\end{cases}
$$

correspond to the order of the virtual MIMO channel, and $\underline{\mathbf{H}}_{l}^{(k)}$ is the channel matrix of the lth virtual tap. Vector

$$
\underline{\mathbf{n}}^{(k)} \triangleq\left[\underline{\mathbf{n}}_{0}^{(k)^{\top}}, \cdots, \underline{\mathbf{n}}_{T-1}^{(k)^{\top}}\right]^{\top} \in \mathbb{C}^{k M_{\mathrm{D}} T},
$$

where

$$
\underline{\mathbf{n}}_{i}^{(k)} \triangleq\left[\mathbf{n}_{i}^{(1)^{\top}}, \cdots, \mathbf{n}_{i}^{(k)^{\top}}\right]^{\top}
$$

$\sim \mathcal{N}\left(\mathbf{0}_{k M_{\mathrm{D}} \times 1}, \sigma^{2} \mathbf{I}_{k M_{\mathrm{D}}}\right)$ denotes the thermal noise present in the $k$-slot equivalent MIMO communication system. Note that the block circulant matrix $\underline{\mathcal{H}}^{(k)}$ can be block diagonalized in a Fourier basis as

$$
\underline{\mathcal{H}}^{(k)}=\mathbf{U}_{T, k M_{\mathrm{D}}}^{\mathrm{H}} \underline{\boldsymbol{\Lambda}}^{(k)} \mathbf{U}_{T, M_{\mathrm{S}}}
$$

Therefore, applying the DFT $\mathbf{U}_{T, k M_{\mathrm{D}}}$ on the $k$-slot received block signal vector (4) yields the following frequency domain block communication model,

$$
\underline{\mathbf{y}}_{f}^{(k)}=\underline{\boldsymbol{\Lambda}}^{(k)} \mathbf{s}_{f}+\underline{\mathbf{n}}_{f}^{(k)},
$$

where

$$
\left\{\begin{array}{l}
\underline{\boldsymbol{\Lambda}}^{(k)} \triangleq \operatorname{diag}\left\{\underline{\boldsymbol{\Lambda}}_{0}^{(k)}, \cdots, \underline{\boldsymbol{\Lambda}}_{T-1}^{(k)}\right\} \in \mathbb{C}^{k M_{\mathrm{D}} \times M_{\mathrm{S}} T}, \\
\underline{\boldsymbol{\Lambda}}_{i}^{(k)}=\sum_{l=0}^{L-1} \underline{\mathbf{H}}_{l}^{(k)} e^{-j(2 \pi i l / T)} \in \mathbb{C}^{k M_{\mathrm{D}} \times M_{\mathrm{S}}} .
\end{array}\right.
$$

In the following, we eliminate the slot index $k$ for notation simplicity. Remember that in fixed-relay based systems, the following data packet processing is performed just one time when $k=K$, while in cooperative $\mathrm{ARQ}$, it is performed each time slot $k$. Let $\tilde{\mathbf{s}}$ denote the conditional estimate of $\mathbf{s}$, and $\sigma_{t, i}^{2}$ the conditional variance of $s_{t, i}$. With the aid of multislot block communication model (12), the MMSE estimate $\mathbf{z}_{f}$ about $\mathbf{s}_{f}$, can be expressed according to the following forward-backward filtering structure,

$$
\mathbf{z}_{f}=\boldsymbol{\Phi} \underline{\mathbf{y}}_{f}-\mathbf{\Psi} \tilde{\mathbf{s}}_{f},
$$

where $\boldsymbol{\Phi}=\operatorname{diag}\left\{\boldsymbol{\Phi}_{0}, \cdots, \boldsymbol{\Phi}_{T-1}\right\}$ is the multi-slot forward MMSE filter given by,

$$
\left\{\begin{array}{l}
\mathbf{\Phi}_{i} \triangleq \underline{\boldsymbol{\Lambda}}_{i}^{\mathrm{H}} \mathbf{B}_{i}^{-1}, \\
\mathbf{B}_{i}=\sigma^{2} \mathbf{I}_{k M_{D}}+\underline{\boldsymbol{\Lambda}}_{i} \tilde{\Xi} \underline{\boldsymbol{\Lambda}}_{i}^{\mathrm{H}} .
\end{array}\right.
$$

$\tilde{\Xi}$ is an unconditional covariance computed as the time average of conditional covariance matrices $\boldsymbol{\Xi}_{i}$ defined as,

$$
\boldsymbol{\Xi}_{i} \triangleq \operatorname{diag}\left\{\sigma_{1, i}^{2}, \cdots, \sigma_{M_{S}, i}^{2}\right\},
$$

and $\boldsymbol{\Psi}=\operatorname{diag}\left\{\boldsymbol{\Psi}_{0}, \cdots, \boldsymbol{\Psi}_{T-1}\right\}$ is the multi-slot backward MMSE filter given by,

$$
\left\{\begin{array}{l}
\boldsymbol{\Psi}_{i} \triangleq \boldsymbol{\Phi}_{i} \underline{\boldsymbol{\Lambda}}_{i}-\boldsymbol{\Upsilon} \\
\boldsymbol{\Upsilon}=\frac{1}{T} \sum_{i=0}^{T-1} \boldsymbol{\Phi}_{i} \underline{\boldsymbol{\Lambda}}_{i}
\end{array}\right.
$$

After computing (14), the inverse DFT (IDFT) is then applied to $\mathbf{z}_{f}$ to obtain the equalized time domain sequence,

$$
\mathbf{z}=\mathbf{U}_{T, M}^{\mathrm{H}} \mathbf{z}_{f} .
$$

The MMSE estimate $z_{t, i}$ corresponding to antenna $t$ and channel use $i$ can be simply extracted from $\mathbf{z}$ as $z_{t, i}=\mathbf{e}_{t, i}^{\mathrm{H}} \mathbf{z}$, with $\mathbf{e}_{t, i}$ denotes the $\left(M_{S} i+t\right)$ th vector of the canonical basis. At the $n$th iteration, The extrinsic LLRs values $\phi_{t, i, m, n}^{(e)}$ corresponding to coded and interleaved bits $b_{t, i, m}$ are then produced using the max-log simplification,

$$
\begin{gathered}
\phi_{t, i, m, n}^{(e)}=\min _{s \in \mathcal{S}_{0}^{m}}\left\{\frac{\left|z_{t, i}-g_{t, i} s\right|^{2}}{\theta_{t, i}^{2}}-\sum_{j \neq m} \phi_{t, i, j, n}^{(a)} \lambda_{j}\{s\}\right\} \\
-\min _{s \in \mathcal{S}_{1}^{m}}\left\{\frac{\left|z_{t, i}-g_{t, i} s\right|^{2}}{\theta_{t, i}^{2}}-\sum_{j \neq m} \phi_{t, i, j, n}^{(a)} \lambda_{j}\{s\}\right\},
\end{gathered}
$$

where $g_{t, i}$ and $\theta_{t, i}^{2}$ denote, respectively, the equivalent channel gain at the output of equalizer and the residual interference variance corresponding to discrete time $i$, and transmit antenna $t, \phi_{t, i, j, n}^{(a)}$ is the a-priori LLR for coded bit $b_{t, i, j}$ obtained from the decoder in the previous iteration, $\lambda_{j}\{s\}$ is an operator extracting the $j$ th bit labeling the symbol $s \in \mathcal{S}$ and $\mathcal{S}_{\beta}^{m}$ is the set of symbols having the $m$ th bit set to $\beta$, i.e., $\mathcal{S}_{\beta}^{m}=$ $\left\{s: \lambda_{m}\{s\}=\beta\right\}$. The calculated extrinsic LLRs are then deinterleaved and fed back to the soft-input-soft-output (SISO) decoder.

\section{B. An Efficient Implementation Scheme For Relay-Assisted System With $K M_{D}>M_{S}$}

Note that the proposed packet combining approach requires the computation of matrix inverses $\mathbf{B}_{0}^{-1}, \cdots, \mathbf{B}_{T-1}^{-1} \in$ $\mathbb{C}^{k M_{D} \times k M_{D}}$ at each turbo iteration, which involve a complexity order cubic against $k M_{D}$. Furthermore, the signals received at slots $1, \cdots, k$ and their corresponding CFRs have to be stored at the receiver. The implementation of such a receiver could be feasible if $K M_{D} \leq M_{S}$. However, if $K M_{D}>M_{S}$, this packet combining approach could not be feasible in practice anymore, since the receiver will require a huge memory that increase linearly with the number of relays as well as the inversion of large matrices. In that case, to prevent the computation of $k M_{D} \times k M_{D}$ matrix inversions, we apply the matrix inversion lemma [13] that allow us to express the inverse of $\mathbf{B}_{i}^{-1}$ as

$$
\mathbf{B}_{i}^{-1}=\frac{1}{\sigma^{2}}\left\{\mathbf{I}_{k M_{D}}-\underline{\boldsymbol{\Lambda}}_{i} \mathbf{C}_{i}^{-1} \underline{\boldsymbol{\Lambda}}_{i}^{\mathrm{H}}\right\},
$$

with $\mathbf{C}_{i}=\sigma^{2} \tilde{\boldsymbol{\Xi}}^{-1}+\underline{\boldsymbol{\Lambda}}_{i}^{H} \underline{\boldsymbol{\Lambda}}_{i} \in \mathbb{C}^{M_{S} \times M_{S}}$. This reduces the implementation cost since the complexity order becomes cubic against $M_{S}$ instead of $k M_{D}$. For the memory size issue, we 
introduce two variables recursively computed, $\underline{\tilde{\mathbf{y}}}_{f}^{(k)}$ and $\underline{\mathbf{D}}_{i}^{(k)}$, to avoid storing received signals and CFRs corresponding to all time slots. The first variable $\underline{\tilde{\mathbf{y}}}_{f}^{(k)}$ is introduced to store the received signals and calculated using the following recursion,

$$
\left\{\begin{array}{l}
\tilde{\mathbf{y}}_{f}^{(k)}=\tilde{\mathbf{y}}_{f}^{(k-1)}+\boldsymbol{\Lambda}^{(k)^{H}} \mathbf{y}_{f}^{(k)}, \\
\tilde{\mathbf{y}}_{f}^{(0)}=\mathbf{0}_{T M_{S} \times 1} .
\end{array}\right.
$$

The second variable $\underline{\mathbf{D}}_{i}^{(k)}$ is used to store the channel frequency responses and calculated as,

$$
\left\{\begin{array}{l}
\underline{\mathbf{D}}_{i}^{(k)}=\underline{\mathbf{D}}_{i}^{(k-1)}+\boldsymbol{\Lambda}_{i}^{(k)^{H}} \boldsymbol{\Lambda}_{i}^{(k)}, \\
\underline{\mathbf{D}}_{i}^{(0)}=\mathbf{0}_{M_{S} \times M_{S}} .
\end{array}\right.
$$

Note that the storage requirements become linear in term of transmit antennas and insensitive to the receive antennas and the number of relays. By using the matrix inversion lemma, we re-write the expression of soft MMSE packet combining as,

$$
\mathbf{z}_{f}=\Gamma_{\tilde{\mathbf{y}}_{f}^{(k)}}^{(\boldsymbol{n}}-\boldsymbol{\Omega} \tilde{\mathbf{s}}_{f},
$$

where $\boldsymbol{\Gamma}=\operatorname{diag}\left\{\boldsymbol{\Gamma}_{0}, \cdots, \boldsymbol{\Gamma}_{T-1}\right\} \in \mathbb{C}^{T M_{S} \times T M_{S}}$, and $\boldsymbol{\Omega}=$ $\operatorname{diag}\left\{\boldsymbol{\Omega}_{0}, \cdots, \boldsymbol{\Omega}_{T-1}\right\} \in \mathbb{C}^{T M_{S} \times T M_{S}}$ denote the new forward and backward filters, respectively, and are given by,

$$
\begin{aligned}
& \left\{\begin{array}{l}
\boldsymbol{\Gamma}_{i} \triangleq \frac{1}{\sigma^{2}}\left\{\mathbf{I}_{M_{S}}-\underline{\mathbf{D}}_{i}^{(k)} \mathbf{C}_{i}^{-1}\right\}, \\
\mathbf{C}_{i}=\sigma^{2} \tilde{\boldsymbol{\Xi}}^{-1}+\underline{\mathbf{D}}_{i}^{(k)},
\end{array}\right. \\
& \left\{\begin{array}{l}
\boldsymbol{\Omega}_{i} \triangleq \boldsymbol{\Gamma}_{i} \underline{\mathbf{D}}_{i}^{(k)}-\boldsymbol{\Upsilon}, \\
\boldsymbol{\Upsilon}=\frac{1}{T} \sum_{i=0}^{T-1} \boldsymbol{\Gamma}_{i} \underline{\mathbf{D}}_{i}^{(k)} .
\end{array}\right.
\end{aligned}
$$

\section{AdAptive Packet Combining Algorithm for COOPERATIVE ARQ SYSTEMS}

In cooperative ARQ systems, where the data packet decoding is performed each time slot $k$, the use of the efficient packet combining scheme, proposed in Sub-Section III-B, is not always the best solution to reduce the implementation cost. As we showed before, the proposed packet combining scheme involve the inversion of matrices, and depending on the implementation scheme, this matrices inversion introduces a computational complexity cubic in term of number of transmit antennas or virtual receive antennas. Therefore, if the relayassisted system has the number of receive antennas less than transmit antennas, i.e. $M_{D}<M_{S}$, the use of the first packet combining scheme, proposed in Sub-Section III-A, during the first slots, enable to reduce the computational complexity of the receiver. However, when the receiver fails to correctly decode the data packet before the number of virtual receive antennas, in the next slot $k$, becomes greater than transmit antennas, i.e. $k M_{D}>M_{S}$, the first packet combining scheme is not the best implementation way to reduce the computational complexity anymore. In that case, the efficient packet combining scheme, proposed in Sub-Section III-B, becomes the best solution. To take advantage of both packet combining scheme proposed in this paper, we introduce an adaptive turbo packet combining algorithm for cooperative ARQ that we summarize in Table I.
Table I

Summary Of The Adaptive Turbo Packet Combining Algorithm FOR COOPERATIVE ARQ SYSTEM

0. $\quad$ At each round $k$

Compare $k M_{D}$ and $M_{S}$

1. If $k M_{D} \leq M_{S}$

1.1. Construct $\underline{\mathbf{y}}_{0}^{(k)}, \cdots, \underline{\mathbf{y}}_{T-1}^{(k)}$ and $\underline{\mathbf{H}}_{0}^{(\mathrm{k})}, \cdots, \underline{\mathbf{H}}_{L-1}^{(\mathrm{k})}$ using (5) and (8), respectively.

1.2. Compute the DFT of the virtual received signal and the CFRs at slot $k$, i.e. $\underline{\mathbf{y}}_{f}^{(k)}$ and $\underline{\Lambda}^{(k)}$, respectively.

1.3. For each iteration,

1.3.1. Compute the forward and backward filters using (15) and (17).

1.3.2. Compute the MMSE estimate of $\mathbf{x}_{f}$ using (14).

1.3.3. Compute the extrinsic LLRs.

1.3.4. Perform SISO decoding.

1.4. end 1.3.

1.5. Frame error test

1.5.1. If "correct frame" then send "ACK" and empty the memory buffers.

Otherwise, compare $(k+1) M_{D}$ and $M_{S}$. If $(k+1) M_{D}>M_{S}$, compute $\underline{\tilde{\mathbf{y}}}_{f}^{(k)}=\underline{\boldsymbol{\Lambda}}^{(k)^{H}} \underline{\mathbf{y}}_{f}^{(k)}$ and $\underline{\mathbf{D}}^{(k)}=\underline{\boldsymbol{\Lambda}}^{(k)^{H}} \underline{\boldsymbol{\Lambda}}^{(k)}$, empty the memory buffers of the old setting, and replace it by $\underline{\mathbf{y}}_{f}^{(k)}$ and $\underline{\mathbf{D}}^{(k)}$.

1.5.2. Go to $\mathbf{0}$.

2. If $k M_{D}>M_{S}$

2.1. Update $\underline{\mathbf{y}}_{f}^{(k)}$ and $\underline{\mathbf{D}}^{(k)}$ using recursions (21) and (22), respectively.

2.2. For each iteration,

2.2.1. Compute the forward and backward filters using (24) and (25).

2.2.2. Compute the MMSE estimate of $\mathbf{x}_{f}$ using (23).

2.2.3. Compute the extrinsic LLRs.

2.2.4. Perform SISO decoding.

2.3. end 2.2.

2.4. Frame error test

2.4.1 If "correct frame" then send "ACK" and empty the memory buffers.

2.5. Go to $\mathbf{0}$. Otherwise, send "NACK".

\section{Performance Evaluation}

In this section, we evaluate the block error rate (BLER) performance of the proposed packet combining scheme. We use conventional LLR-level packet combining as a reference. In this basic combining scheme, the turbo equalization is performed separately for each slot, and before SISO decoding, the extrinsic LLRs are simply added together with those obtained at the last iteration of previous slot. In all simulations, we consider a STBICM scheme where the encoder is a 16 state convolutional code with polynomial generators $(35,23)_{8}$, and the modulation scheme is quadrature phase shift keying (QPSK). The length of the code frame is 2048 bits including tails, and the $\mathrm{CP}$ length is $T_{C P}=3$. We use the MaxLog-MAP algorithm for SISO decoding, and the iterative MMSE receiver at the destination runs three turbo iterations. We consider relay-assisted systems with one, two, and three relays. The $S N R_{\mathrm{SD}}$ appearing in all figures is the $\mathrm{S} \rightarrow \mathrm{D}$ link signal-to-noise ratio per useful bit per receive antenna. For simplicity, we consider a homogeneous case in which the 


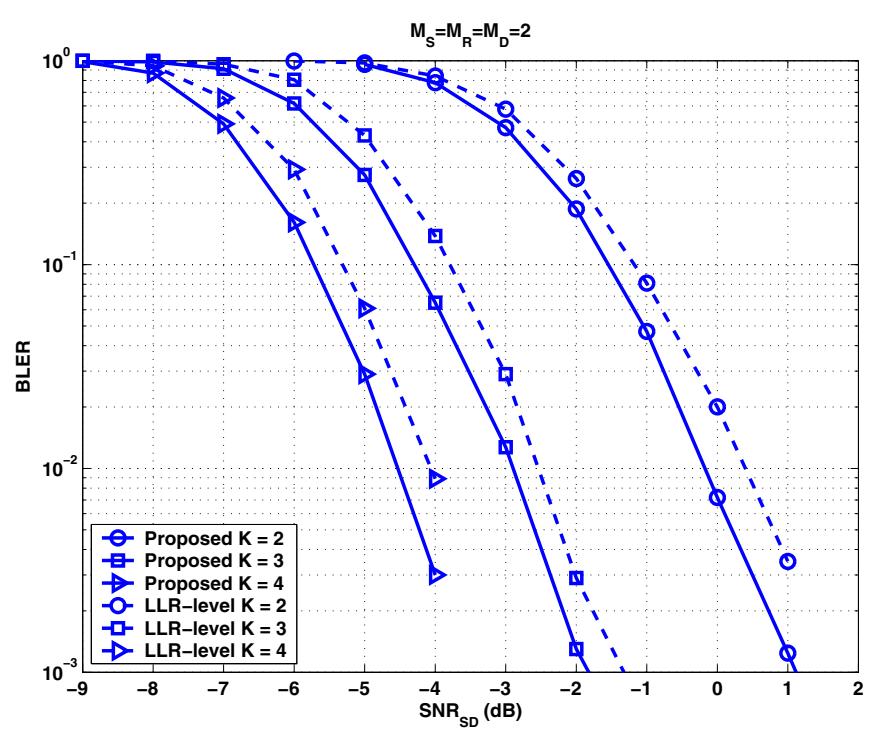

Figure 1. BLER performance for CC $(35,23)_{8}$, QPSK, $M_{\mathrm{S}}=M_{\mathrm{R}}=$ $M_{\mathrm{D}}=2, L=3$ equal energy paths, $l_{S R}=0.3$ and the path loss exponent $\kappa=3$.

distance between the source and the relay $l_{S R}$, the relay and the destination $l_{\mathrm{RD}}$, and the source and the destination $l_{\mathrm{SD}}$ are normalized as $l_{\mathrm{SR}}+l_{\mathrm{RD}}=l_{\mathrm{SD}}=1$. We assume that all relays are at the same distance to the source, i.e. $l_{S R}=0.3$ and the destination, i.e. $l_{\mathrm{RD}}=0.7$. All links have the same frequency-selective fading channel profile, i.e., $L=3$ equal power paths with the same path loss exponent $\kappa=3$. The link average energy is assumed to be $E_{A B}=\left(l_{A B}\right)^{-\kappa}$ with $A=\mathrm{S}$ or $\mathrm{R}$, and $B=\mathrm{R}$ or $\mathrm{D}$. Our main concern is to show the superior performance of the proposed combining strategy compared to LLR-level combining. First, we consider in Fig. 1 a relaying system with the same number of transmit and receive antennas $M_{\mathrm{S}}=M_{\mathrm{R}}=M_{\mathrm{D}}=2$. We observe that the proposed combining strategy clearly outperforms LLRlevel combining. However, the performance gap, at $10^{-2}$ BLER, is less than $1 \mathrm{~dB}$ for systems with one, two and three relays. Fig. 2 shows the performance of an overloaded system where $M_{\mathrm{S}}=M_{\mathrm{R}}=2$ and $M_{\mathrm{D}}=1$. In this case, the proposed combining strategy significantly outperforms LLRlevel combining, i.e., the performance gap is more than $2 \mathrm{~dB}$ at $10^{-2}$ BLER for systems with one relay and $1.5 \mathrm{~dB}$ for systems with three relays.

\section{CONCLUSION}

In this paper, we have proposed turbo packet combining strategies for multi-relay-assisted systems operating over MIMO broadband channel. Using the "virtual antennas" concept, we have introduced a frequency domain MMSE-based turbo packet combining scheme where all slots received signals and their corresponding CFRs are jointly used to decode the data packet. Then, we have provided an efficient recursive implementation for the proposed scheme, and have shown that both its computational complexity and memory requirements are quite insensitive to the number of relays in the system. We have also introduced an adaptive packet combining algorithm that enable to reduce the receiver implementation cost

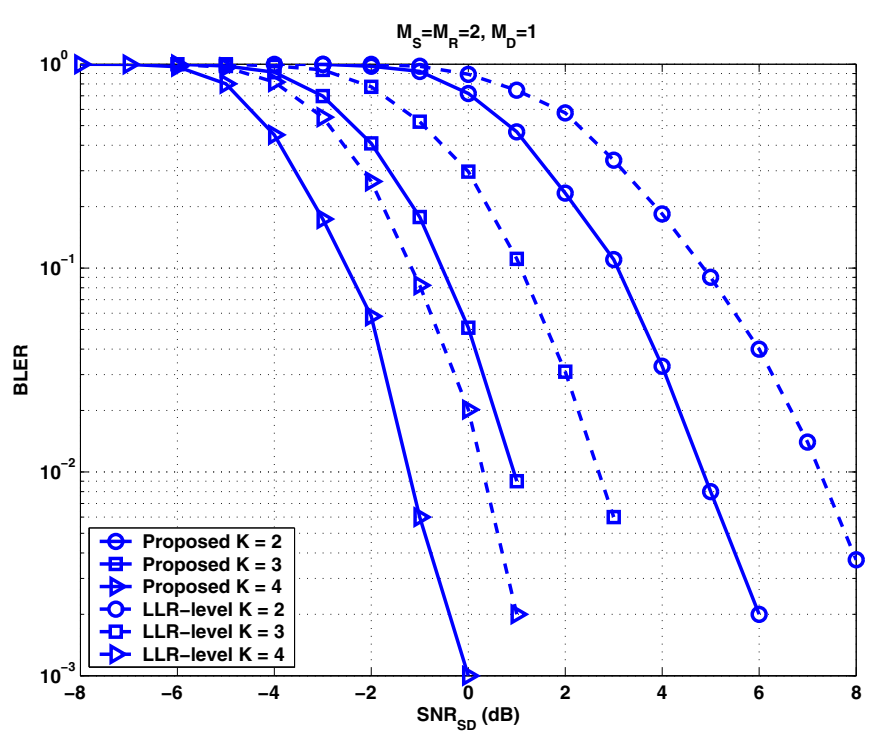

Figure 2. BLER performance for CC $(35,23)_{8}$, QPSK, $M_{\mathrm{S}}=M_{\mathrm{R}}=2$, $M_{\mathrm{D}}=1 L=3$ equal energy paths, $l_{S R}=0.3$ and the path loss exponent $\kappa=3$.

for systems using cooperative ARQ transmission technique. Simulations results have shown that the proposed combining strategy provides better BLER performance than conventional LLR-level combining.

\section{REFERENCES}

[1] J. N. Laneman, G. W. Wornell, and D. N. C. Tse, "An efficient protocol for realizing cooperative diversity in wireless networks," IEEE International Symposium on Information Theory (ISIT), Washington, DC, June 2001.

[2] A. Sendonaris, E. Erkip, and B. Aazhang, "User cooperation diversity Part I \& Part II,” IEEE Trans. Commun., vol. 51, pp. 1927-1948, Nov. 2003.

[3] J. Boyer, D. D. Falconer, and H. Yanikomeroglu, "Multihop diversity in wireless relaying channels," IEEE Trans. on Comm., vol. 52, pp. 18201830, Oct. 2004.

[4] F. Atay Onat, H. Yanikomeroglu, and S. Periyalwar, "Relay-assisted spatial multiplexing in wireless fixed relay networks,", IEEE GLOBECOM, San Francisco, USA, Nov.- Dec. 2006.

[5] Q. Jia, T. Lv, and G. Ping, "An efficient scheme for joint equalization and interference cancellation in distributed cooperative diversity networks", Communication Networks and Services Research (CNSR), Halifax, Canada, May 2008.

[6] R. U. Nabar, F. W. Kneubiihler, and H. Boelcskei, "Performance limits of amplify-and-forward based fading relay channels", IEEE International Conference on Acoustics, Speech, and Signal Processing (ICASSP), Montreal, Canada, May 2004.

[7] H. Mheidat, M. Uysal, and N. Al-Dhahir, "Equalization techniques for distributed space-time block codes with amplify-and-forward relaying," IEEE Trans. Signal Process., vol. 55, pp. 1839-1852, 2007.

[8] H. Xiong and J. X. P. Wang, "Frequency-domain equalization and diversity combining for demodulate-and-forward cooperative systems", IEEE ICASSP, Las Vegas, Nevada, USA, March-April 2008.

[9] T. Ait-Idir, and S. Saoudi, "Turbo packet combining strategies for the MIMO-ISI ARQ channel," IEEE Trans. Commun., In Press.

[10] T. Ait-Idir, H. Chafnaji, and S. Saoudi, "Turbo packet combining for broadband space-time BICM ARQ systems with co-channel interference," Conditionally accepted, IEEE Trans. Wireless Commun.

[11] Y. Zhang, H. H. Chen, and M. Guizani, Cooperative Wireless Communications, Auerbach Publications, 2009.

[12] B. Zhao and M. C. Valenti, "Practical relay networks: A generalization of hybrid-ARQ," IEEE J. Select. Areas. Comm., vol. 23, no. 1, Jan. 2005.

[13] S. Haykin, Adaptive Filter Theory, 3rd Ed. Upper Saddle River, NJ: Prentice-Hall, 1996. 